
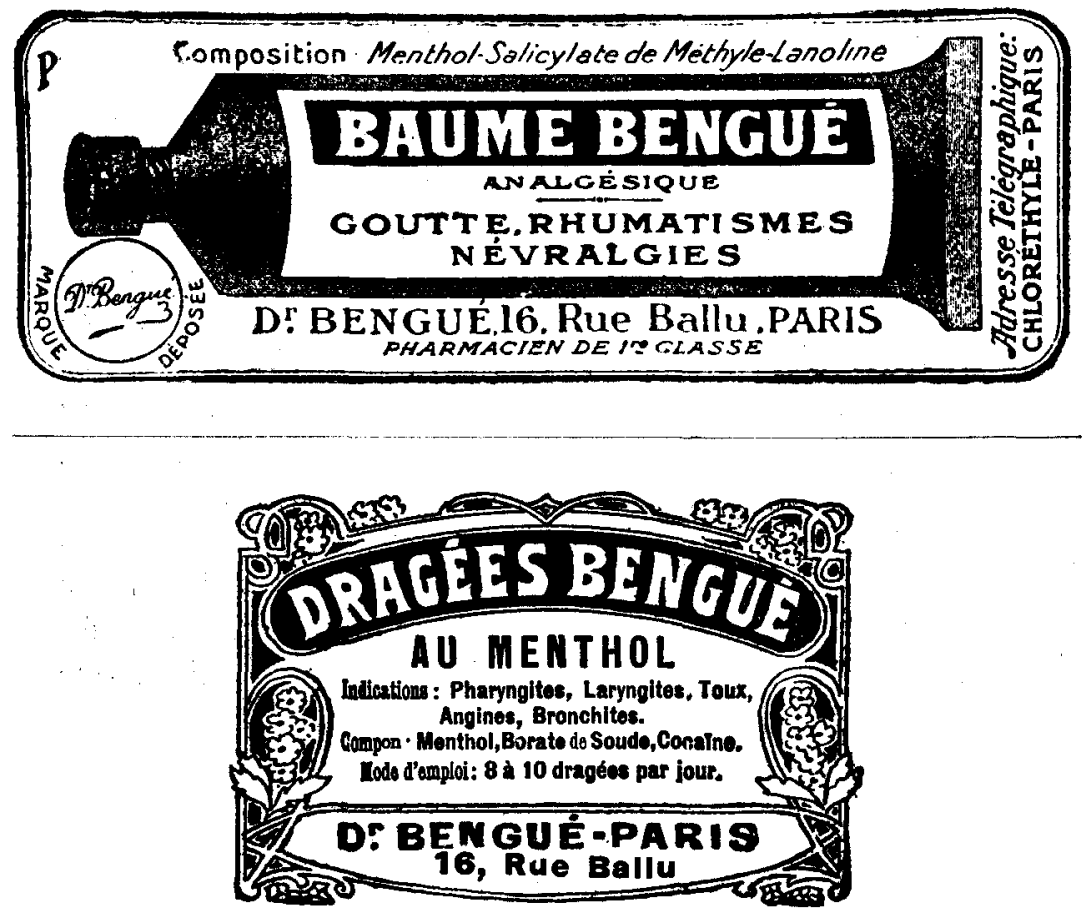

\title{
The World's Children
}

ILLUSTRATED -- FOURPENCE MONTHLY

News, Views and Reviews on the Citizens of To-morrow The English-Speaking Organ of the Declaration of Geneva Edited by EDWARD FULLER

“THE WORLD'S CHILDREN is real good reading”, says SYBIL THORNDIKE

"I rely upon THE WORLD'S CHILDREN for the most useful information and I shall enjoy it to my last days", says M. HENRI ROLLET, the veteran honorary Judge of the Juvenile Court, Paris

Order a copy to-day from your book-seller or, better still, send a year's subscription $5 \mathrm{~s} ., \$ 1.25$, or 6 gold francs to the publishers :

THE WEARDALE PRESS, Ltd., 40, Gordon Square, LONDON W.C. 1 (England) 


\section{Adresses des Comités centraux (suite).}

* BELGIQUE. - Croix-Rouge de Belgique, rue de Livourne, 80, Bruxelles. BOLIVIE. - Croix-Rouge bolivienne, La Paz.

BRESIL. - Croix-Rouge brésilienne, Praça Vieira Souto, 10, Rio de Janeiro. BULGARIE. - Croix-Rouge bulgare, 19, boul. Totleben, Sofia.

CANADA. - Croix-Rouge canadienne, 410, Sherbourne Street, Toronto.

CHILI. - Croix-Rouge chilienne, Calle Catedral 1572, Santiago de Chile.

CHINE. - Croix-Rouge chinoise, Pékin et 26, Kiukiang Road, Shanghaï.

COLOMBIE. - Croix.Rouge colombienne, Bogota.

COSTA-RICA. - Croix-Rouge costaricienne, Calle 5. Sur., San José.

CUBA. - Croix-Rouge cubaine, Ignacio Agramonte et av. de Belgica, La Havane.

DANEMARK. - Croix-Rouge danoise, 2, Ved Stranden 2 Sal, Copenhague, K.

DANTZIG. - Croix-Rouge de Dantzig, Neugarten, 12-16, Dantzig.

REPUBLIQUE DOMINICAINE. - Croix-Rouge dominicaine, Saint-Domingue.

FGYPTE. - Croissant-Ronge égyptien, rue Ismail Pacha, 8 (Garden City), Le Caire.

EQUATEUR. - Croix-Rouge équatorienne, Avenida Columbia, 118, Quito.

ESPAGNE. - Croix-Rouge espagnole, Calle de Sagâsta, 10 (Estafeta, 4), Madrid.

ESTONIE. - Croix-Rouge estonienne, Niguliste Tän, 12, Reval.

ETATS-UNIS. - Croix-Rouge américaine (Central Committee of the American National

Red Cross), National Headquarters, Washington, D.C.

FINLANDE. - Croix-Rouge de Finlande, 16, Annankatu, Helsingfors.

FRANCE. - Croix-Rouge française, rue François-Ier, 21, Paris, 8e.

GRANDE-BRETAGNE. - Croix-Rouge britannique (British Red Cross Society),

14 Grosvenor Crescent, London, S.W.I.

GRECE. - Croix-Rouge hellénique, rue Solon, 39, Athènes.

GUATEMALA. - Croix-Rouge guatémaltèque, 10, Calle Oriente, no 32 . Guatémala.

HONGRIE. - Croix-Rouge hongroise, Baross utca 15, Budapest, VIII.

INDE. - Croix-Rouge de l'Inde (Indian Red Cross Society), Talkatora Road, New-Delhi

(en hiver jusqu'au ler mai); Sheerwood, Simla, w.c. (en été jusqu'au ler novembre).

ISLANDE. - Croix-Rouge islandaise, Reykjavik.

ITALIE. - Croix-Rouge italienne, via Toscana, 12, Rome (25).

JAPON. - Croix-Rouge japonaise, 5 Shiba Park, Tokio.

LETTONIE. - Croix-Rouge lettone, Skolas ielá, 1, Riga.

LITHUANIE. - Croix-Rouge lithuanienne, Kesucio g-vé 8, Kaunas.

LUXEMBOURG. - Croix-Rouge luxembourgeoise, 10, av. Marie-Thérèse, Luxembourg.

MEXIQUE. - Croix-Rouge mexicaine, Avenida Isabel la Católica, 25 Mexico, D.F.

NORVEGE. - Croix-Rouge de Norvège, Grew Wedels Plass 5, Oslo.

NOUVELLE-ZELANDE. - Croix-Rouge néo-zélandaise, P.O.Box 969, Wellington, N.Z.

PANAMA. - Croix-Rouge de Panama, Panama.

PARAGUAY. - Croix-Rouge paraguayenne, Avenida España, 505, Assomption.

** PAYS-BAS. - Croix-Rouge néerlandaise, Princessegracht, 27, La Haye.

PEROU. - Croix-Rouge péruvienne, Lima.

PERSE. - Lion et Soleil-Rouges de Perse, Téhéran.

POLOGNE. - Croix-Rouge polonaise, Smolna, 6, Varsovie.

PORTUGAL. - Croix-Rouge portugaise, Palacio da Rocha do Conde d'Obidos, Lisbonne.

ROUMANIE. - Croix-Rouge roumaine, Strada Biserica Amzei, 29. Bucarest.

SALVADOR. - Croix-Rouge de Salvador, 3a calle Poniente, 21, San Salvador.

SIAM. - Croix-Rouge siamoise, Bangkok.

SUEDE. - Croix-Rouge suédoise (Svenska Röda Korset), Artillerigatan, 6, Stockholm, 14.

SUISSE. - Croix-Rouge suisse, Taubenstrasse, 8, Berne.

TCHECOSLOVAQUIE. - Croix-Rouge tchécoslovaque, Neklanova ul., 147, Prague VI.

TURQUIE. - Croissant-Rouge turc, Ankara.

*** U.R.S.S. (Union des républiques soviétiques socialistes). - Alliance des Sociétés de la

Croix-Rouge et du Croissant-Rouge de l'U.R.S.S., Ipatievsky péréoulok, 6, Moscou. URUGUAY. - Croix-Rouge uruguayenne, Calle 18 de Julio no 2338 , Montevideo.

VENEZUELA. - Croix-Rouge vénézuélienne, Muñoz a Piñangoo, 10, Caracas.

YOUGOSLAVIE. -- Croix-Rouge yougoslave, Simina ulica, 19, Beograd.

\footnotetext{
- Section: Croix-Rouge du Congo, rue de Livourne, 80, Bruxelles.

* Branche: Croix-Rouge des Indes néerlandaises, Veltwreden (Java).

** Membres Croix-Rouge arménienne, Erivan.

de Croissant-Rouge de l'Azerbéidjan, rue Zévine, 1, Batou.

l'Alliance: Croix-Rouge géorgienne, 15, rue Krylov, Tiflis.

Croix-Rouge russe (R.S.F.S.R.), Ilünka 12, Moscou.

Croix-Rouge de la Russie Blanche, 3 , rue Marx, Minsk.

Croissant-Rouge du Tadjikistan, Sialinabad.

Croissant-Rouge du Turkménistan, Achabad.

Croix-Rouge ukranienne, 7 , place du Thêtire, Kharkiv.
} 


\section{Sommaire du Bulletin}

T. LXIII, $\mathbf{N}^{\circ} 362$

OCTOBRE 1932

Comité international. -- Entrée en vigueur de l'Union internationale de secours, 885. - Tremblements de terre en Grèce (communication conjointe $\mathrm{n}^{\circ}$ 16), 888. - Dons pour les victimes dos tremblements de terre en Grèce, 890 .

Ligue. - Tremblements de terre en Grèce, 890. - Revue et Bulletin, 891 .

Belgique. - Rapport de la Croix-Rouge du Congo sur l'année 1931, 891.

Danemark. - L'ouvre sanitaire et sociale de la Croix-Rouge danoise en 1931,892 .

France. - Activité de l'Union des femmes de France en Afrique du Nord, 894.

Italie. - Présidence de la Croix-Rouge italienne, 895. — Secrétariat international d'informations privées, 896 .

Paraguay. - Mobilisation de la Croix-Rouge, 896.

Pays-Bas. - Ratification des Conventions conclues à Genève le 27 juillet 1929, 897.

Pologne. - Activité de la Croix-Rouge polonaise en 1931, 898.

Suède. - L'œuvre sanitaire et sociale de la Croix-Rouge suédoise, 901.

Tadjikistan. - L'astivité du Croissant-Rouge du Tadjikistan en 1931, 905.

Tchécoslovaquie. - Trêve de la Croix-Rouge, 905.

Vénézuéla. - Comité central de la Croix-Rouge vénézuélienne, 906.

La Revue internationale de la Croix-Rouge, Bulletin international des Sociétés de la Croix-Rouge, paraît à la fin de chaque mois.

Prix, franco, un an: Fr. 20.- Le numéro: Fr. 2.Années 1919, 1920 et 1921 (rares): Fr. 30.Administration : Promenade du Pin, 1, Genève. 